\title{
Validation and Initial Application of a Semiautomatic Aneurysm Measurement Software: A Tool for Assessing Volumetric Packing Attenuation
}

\author{
H. Takao, T. Ishibashi, T. Saguchi, H. Arakawa, M. Ebara, K. Irie, and Y. Murayama
}

\begin{abstract}
BACKGROUND AND PURPOSE: Precise aneurysm measurements and volume embolization ratios are essential for long-term durability of endovascular coil embolization. We evaluated the accuracy of newly developed semiautomatic cerebral aneurysm measurement software, NeuroVision, and explored the value of volume embolization ratio in the prediction of re-treatment.
\end{abstract}

MATERIALS AND METHODS: We compared software-derived volume measurements of 4 silicone aneurysm models with those calculated with an approximation formula and ground truth values (validation study). We used NeuroVision to retrospectively evaluate outcomes of 100 unruptured aneurysms (97 patients) treated with embolization (clinical study). Aneurysm size (height, width, and neck), volume, and volume embolization ratios were calculated for 3 groups (stable, recanalization, and re-treatment) and were compared.

RESULTS: This validation study illustrated higher accuracy of NeuroVision in computing aneurysm volume compared with an approximation formula: percentage absolute errors were $4.50 \% \pm 3.18 \%$ and $23.07 \% \pm 17.60 \%$, with maximal percentage absolute errors of $8.99 \%$ and $45.63 \%$, respectively. Of 100 unruptured aneurysms, 20 recanalized and 12 were re-treated. Average volume embolization ratios of stable and re-treated aneurysms were $24.88 \% \pm 5.91 \%$ and $20.50 \% \pm 4.06 \%$, respectively $(P \leq .01)$. The optimal volume embolization ratio cutoff point for re-treatment was $<19.15 \%$, at which the Youden index was 0.50 (sensitivity, $58.33 \%$; specificity, $87.50 \%$; area under the receiver operating characteristic curve, 0.74).

CONCLUSIONS: The NeuroVision software provided accurate aneurysm volume measurements and may be a useful standardized tool to measure aneurysm size and volume, especially for multicenter clinical studies. Volume embolization ratio may be a valuable predictor of aneurysm occlusion changes.

ABBREVIATIONS: AF = approximation formula; $\mathrm{GDC}=$ Guglielmi detachable coils; NV = NeuroVision; ROC = receiver operating characteristic; VER = volume embolization ratio

E

ndovascular coil embolization of cerebral aneurysms is now considered a valuable method to safely and effectively prevent aneurysm rupture. ${ }^{1-4}$ However, immediate postembolization angiographic evidence of complete aneurysm occlusion does not guarantee a good long-term outcome. ${ }^{5}$ Volume embolization ratio (VER), the percentage of aneurysm sac volume occupied by coils, is considered a valuable predictor of aneurysm recanalization, ${ }^{6-11}$ in addition to large size, wide neck, ruptured status, and suboptimal immediate angiographic result.

However, the accuracy of the methods used to measure aneu-

Received April 1, 2013; accepted after revision August 26

From the Division of Endovascular Neurosurgery and Neurosurgery (H.T., T.I., H.A., M.E., K.I., Y.M.), Jikei University School of Medicine, Tokyo, Japan; and Department of Neurosurgery (T.S.), Japanese Red Cross Medical Center, Tokyo, Japan.

Please address correspondence to Yuichi Murayama, MD, Division of Endovascular Neurosurgery, Department of Neurosurgery, Jikei University School of Medicine, 3-25-8 Nishi-Shinbashi, Minato-ku, Tokyo, Japan; e-mail: ymurayama@jikei.ac.jp

- Indicates open access to non-subscribers at www.ajnr.org

http://dx.doi.org/10.3174/ajnr.A3777 rysm volume, and thus assess these predictors of recanalization, has not been well evaluated. A standardized aneurysm measurement methodology is necessary to enable multiple institutions to conduct collaborative investigations and compare their results.

A new analytic measurement software was developed for the measurement of cerebral aneurysms (NeuroVision [NV]; Cybernet Systems, Tokyo, Japan). We evaluated the accuracy of this software by using phantom aneurysm models. In addition, we used the software to retrospectively analyze the long-term outcome of a series of embolized aneurysms and investigate the value of VER, as semiautomatically computed by NV, in the prediction of aneurysm recurrence and re-treatment.

\section{MATERIALS AND METHODS Software Description and In Vitro Validation}

NV Software. The NV software measurement process was initiated by a few discrete steps (Fig 1). Through a simple user interface, the image region containing the aneurysm was separated 

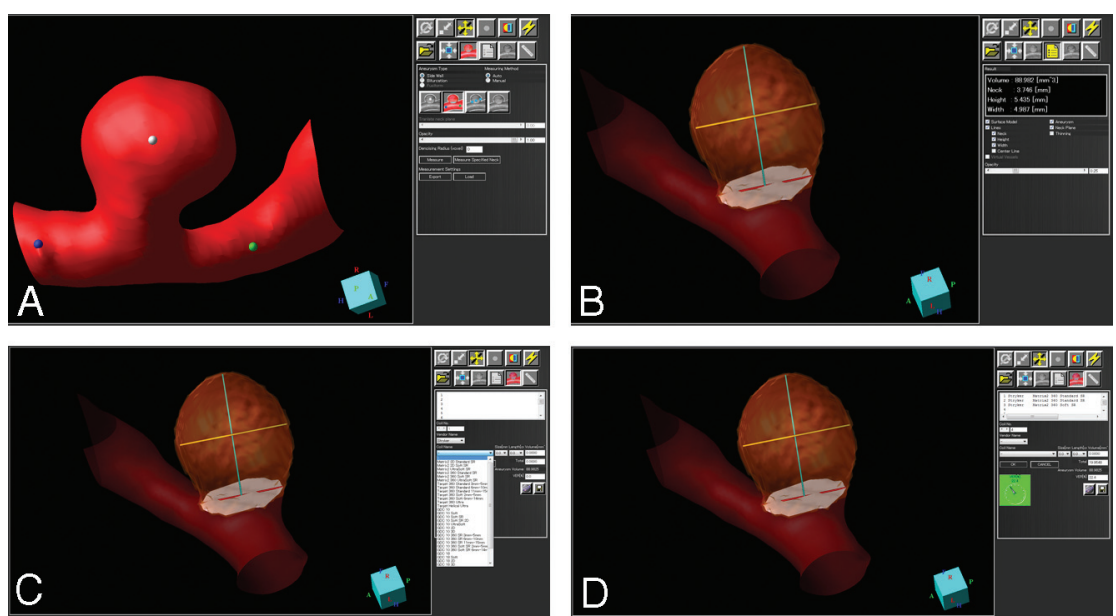

FIG 1. A, A white marker in the aneurysm and 2 markers (blue and green) in the parent artery are needed to start the measurements in NV. The markers are easily placed manually by the user. $B$, Height, width, neck length, and aneurysm volume are measured semiautomatically with NV. C, To measure VER, we select the type of GDC coils from the drop-down menu. $D$, Aneurysm VER is displayed on a green-colored meter on the right.
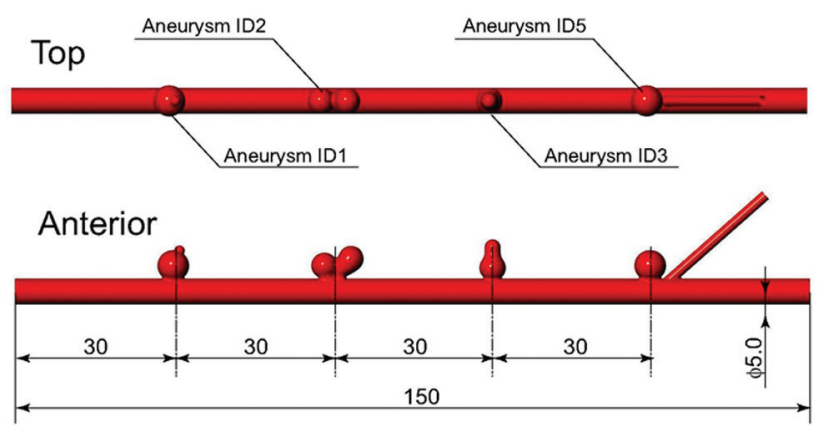
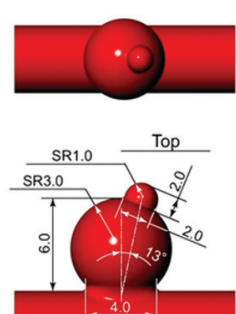

Anterior

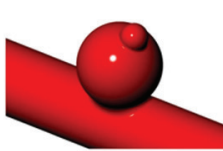

Perspective

Aeurysm1 $\left(\begin{array}{l}6 \times 6, \text { neck: } 4.0 \\ 2 \times 2, \text { neck: } 2.0\end{array}\right)$ Volume: $120.0 \mathrm{~mm}^{3}$

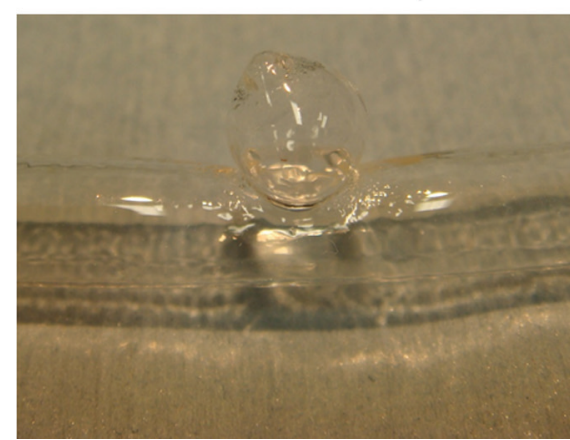

FIG 2. Silicone models.

manually, and an image threshold was subsequently applied to extract the aneurysm and blood vessels from the surrounding background image. The threshold was semiautomatically set by use of the discriminant analysis method, which guaranteed that the selected threshold optimally separated the image intensities belonging to blood vessels from those that belonged to the background. ${ }^{12}$ The optimal threshold was that which corresponded to the maximal value of the Fisher linear discriminant, defined as the ratio of variance between the image intensities of the 2 classes (vessel and background) to the within-class variance. ${ }^{13}$ Use of discriminant analysis to determine the image segmentation threshold avoided the problems associated with varying measurements because of the arbitrary choice of window center and with the varying image quality of different imaging systems.
Aneurysm Size Measurements. Neck length, height, and width were measured by NV. Aneurysm neck length was defined as the largest diameter at the orifice plane of the aneurysm. Aneurysm height was defined as the longest distance from the midpoint of the aneurysm neck length line to the aneurysm wall. Aneurysm width was defined as the largest aneurysm diameter perpendicular to the height line.

Aneurysm Volume Measurement. NV virtually reconstructed the parent artery after semiautomatic aneurysm removal. The aneurysm neck plane was manually adjusted for complex aneurysm neck shapes through the manipulation of 3 points on the plane. The aneurysm volume was calculated semiautomatically based on the aneurysm neck plane.

Validation Study. We prepared 4 silicone aneurysm models with known sizes and volumes. The aneurysm models had different shapes: round, with a bleb; conjoined aneurysms (heart-shaped); aneurysm with a daughter sac; and round without bleb (Fig 2).

We compared the ground truth aneurysm volumes with the NV volume measurements and the estimated volumes based on a commonly used approximation formula (AF): [aneurysm volume $=(4 \pi / 3) \times\left(\mathrm{d}_{1} / 2\right) \times\left(\mathrm{d}_{2} / 2\right) \times$ $\left(d_{3} / 2\right)$ ], where $d_{1}, d_{2}$, and $d_{3}$ are 3 orthogonal measurements denoting aneurysm height, width, and breadth. ${ }^{14}$ We calculated the errors of each approach.

Calculation of VER with Use of NV. Coil volume was easily calculated based on the Guglielmi detachable coil (GDC) diameter and length data provided by the manufacturer (Boston Scientific, Natick, Massachusetts), which were preloaded into the NV software. VER was semiautomatically calculated based on the aneurysm volume and the volume of coils used during embolization.

\section{Initial Testing of NV Software in a Clinical Cohort}

Patient Population. To assess the value of the measurement parameters provided by the software, we used NV to retrospectively measure aneurysm volume and VER on 3D DSA images from a cohort selected from our institutional data base of patients with cerebral aneurysms who were treated with coil embolization. A total of 295 patients with 308 cerebral saccular aneurysms underwent endovascular embolization with detachable platinum coils at our institution between January 2006 and August 2008. They included 115 patients with 121 unruptured cerebral aneurysms 
Table 1: Location of 100 unruptured aneurysms treated with GDC

\begin{tabular}{lc}
\hline \multicolumn{1}{c}{ Location } & Number \\
\hline ACA & \\
AcomA & 19 \\
Others & 3 \\
ICA & \\
Top & 6 \\
Anterior choroidal & 5 \\
PcomA & 23 \\
Paraclinoid & 11 \\
Ophthalmic & 6 \\
Cavernous & 3 \\
MCA & 12 \\
Vertebrobasilar system & \\
Tip & 5 \\
SCA & 5 \\
Trunk & 1 \\
PICA & 1 \\
Total & 100 \\
\hline Note-ACA ind and
\end{tabular}

Note:-ACA indicates anterior cerebral artery; AcomA, anterior communicating artery; PcomA, posterior communicating artery; SCA, superior cerebellar artery.

treated only with GDC; only patients treated with GDC $^{3,11}$ were included in this study because only volume information for this coil was available from the manufacturer. Eighteen of the 115 patients were excluded for either refusing to undergo follow-up angiography or only undergoing MRA follow-up (follow-up rate, $84.35 \%$ ). Thus, our study cohort included 97 patients with 100 aneurysms treated with GDC who underwent follow-up angiography approximately 1 year after the procedure. There were 72 women and 25 men. The mean age of the patients at the time of treatment was $58.85 \pm 11.57$ years (age range, $31-83$ years). Seventy-eight $(78.0 \%)$ aneurysms were located in the anterior circulation and $12(12.0 \%)$ in the posterior circulation (Table 1$)$.

Embolization of Aneurysms. Before embolization, patients underwent 3D DSA by use of a biplane flat detector C-arm angiographic system (Axiom Artis dBA; Siemens, Erlangen, Germany). 3D DSA images were reconstructed on a clinical workstation (syngo XWP VB15B; Siemens) at $256 \times 256$ image matrix size.

The embolization procedure was performed with the patient under general anesthesia. Systemic heparinization was administered as an initial 3000-U bolus, followed by $1000 \mathrm{U} /$ hour under whole-blood activated clotting time monitoring throughout the procedure. In all patients, systemic heparinization was continued for 24 hours after embolization.

Aneurysms were embolized with GDC coils by packing as densely as possible. The percentage of occlusion at the end of the procedure was evaluated by embolized volume. VER was calculated by use of the following formula: VER $=$ (volume of the embolization coils)/(volume of the aneurysm $) \times 100$.

Using the classification schema reported by Raymond et $\mathrm{al}^{15}$ and Roy et $\mathrm{al},{ }^{16}$ we categorized the rate of occlusion immediately after initial treatment as measured on angiographic images as: complete occlusion, when the sac and neck were densely packed in any projection; subtotal occlusion (residual neck), when the sac was occluded but there was suspicion of a neck remnant or there was an obvious tiny neck remnant; and partial occlusion (residual aneurysm), when there was loose packing and/or persistent opacification of the sac or neck remnant.

Angiographic Follow-Up Studies. Patients were scheduled for fol- low-up angiography at 1 year after treatment. For the evaluation of follow-up angiographies, the Raymond et al ${ }^{15,16}$ definition of aneurysm recanalization was modified. An aneurysm was classified as stable at follow-up if the degree of occlusion was unchanged or had improved (such as further thrombosis) compared with the end-of-treatment study. Recanalization was defined as an increase in the amount of contrast material filling the aneurysm compared with the angiographic appearance at the end of the treatment, and such an aneurysm was classified as unstable because of coil compaction and aneurysm growth.

Using these definitions, we further classified the follow-up results into 3 groups: stable group, including unchanged occlusion or further thrombosis; recanalization group, including any recanalization; and re-treatment group, including recanalization with re-treatment. Aneurysms that had contrast filling at the neck of $>15 \%$ of the aneurysm height were selected for re-treatment.

Aneurysm and VER Measurement Protocols. Radiology technologists used NV to obtain aneurysm size (millimeters), aneurysm volume (millimeters cubed), and VER (percentage) measurements for all 100 aneurysms. Two neurosurgeons who participated in the coil embolization of these aneurysms reviewed the NV measurements for accuracy.

We then compared the VERs of each group: stable group, recanalization group, and re-treatment group. We further divided the aneurysms into 2 groups according to volume and compared the VERs: The large-volume group included aneurysms with a volume of $>200 \mathrm{~mm}^{3}$, and the small-volume group included aneurysms with a volume of $<200 \mathrm{~mm}^{3}$.

We assessed the impact of aneurysm volume and VER on the probability of recurrence and re-treatment for all groups.

Statistical Analysis. All values were expressed as mean \pm standard deviation. The statistical significance of differences in mean value between 2 populations was assessed by use of the Student $t$ test if variances were equal, as determined by $F$ value; otherwise, the Welch $t$ test was used. If population distribution was not a "normal" distribution, we analyzed it by using the nonparametric Mann-Whitney $U$ test.

A receiver operating characteristic (ROC) curve was used to analyze the effect of varying VER, as a discriminating variable, on embolization outcome (eg, re-treatment vs no re-treatment). The ROC area under the curve (and its $95 \% \mathrm{CI}$ ) was calculated to determine the discriminating level of VER.

The Youden index ${ }^{17,18}(\mathrm{~J})$ was defined as the difference between the true-positive rate and the false-positive rate in the outcome variable. Maximizing this index allowed us to find, from the ROC curve, an optimal cutoff point, independently from the prevalence. J was defined as the maximal vertical distance between the ROC curve and the diagonal or chance line, and it was calculated as $\mathrm{J}=$ maximum ( this measure, the cutoff point on the ROC curve was selected so that it corresponded to the maximal J.

\section{RESULTS}

Validation Study: Silicone Aneurysm Models (AF, NV)

When we compared the accuracy of an AF with that of NV at measuring volume in the 4 silicone aneurysm models, the per- 
Table 2: Comparison of aneurysm volume using AF and NV to ground truth volumes for silicone aneurysm models

\begin{tabular}{clccc}
\hline $\begin{array}{c}\text { Model } \\
\left(\mathbf{m m}^{\mathbf{3}}\right)\end{array}$ & $\begin{array}{c}\text { Type of } \\
\text { Model }\end{array}$ & $\begin{array}{c}\text { Real } \\
\text { Volume }\end{array}$ & Volume 1 & Volume 2 \\
\hline 1 & With bleb & 120.00 & 150.72 & 118.00 \\
2 & Heart & 161.70 & 87.92 & 168.60 \\
3 & Two-ball & 89.00 & 104.67 & 97.00 \\
4 & Ball & 117.10 & 113.04 & 120.70 \\
\hline
\end{tabular}

Note:-Volume 1 using approximation formula (AF). Volume 2 using NeuroVision (NV) software.

Table 3: Percentage absolute error for AF and NV

\begin{tabular}{ccc}
\hline Percentage Absolute Error & AF (\%) & NV (\%) \\
\hline Average & 23.07 & 4.50 \\
Maximum & 45.63 & 8.99 \\
SD & 17.60 & 3.18 \\
\hline
\end{tabular}

Note:-SD indicates standard deviation.

centage absolute error for an AF was $23.07 \% \pm 17.60 \%$ and for NV $4.50 \% \pm 3.18 \%$, with a maximal percentage absolute error of $45.63 \%$ and $8.99 \%$, respectively (Table 2 ; Table 3 ).

\section{Clinical Study (NV)}

Using NV, we calculated size and volume measurements for all aneurysms within 1 minute after data input. Mean aneurysm size measurements were height, $6.93 \pm 3.35 \mathrm{~mm}$; width, $6.07 \pm 2.90$ $\mathrm{mm}$; and neck length, $5.48 \pm 2.27 \mathrm{~mm}$. Mean aneurysm volume was $280.7 \pm 530.6 \mathrm{~mm}^{3}$, and mean VER was $24.13 \% \pm 5.75 \%$.

The VERs by aneurysm location were ICA $(n=54), 24.63 \% \pm$ $5.92 \%$; MCA $(n=12), 22.22 \% \pm 7.25 \%$; anterior cerebral artery $(n=22), 23.80 \% \pm 5.27 \%$; and vertebrobasilar system $(n=12)$, $24.35 \% \pm 4.16 \%$. There was no statistically significant difference in VER among aneurysms at different locations.

\section{Relationship between Immediate Angiographic Occlusion and VER}

Immediate anatomic outcome on 3D angiography was complete occlusion in 17 aneurysms (17\%), residual neck in 78 aneurysms (78\%), and residual aneurysm in 5 aneurysms (5\%). VER in completely occluded aneurysms (large-volume group, $n=1$; smallvolume group, $n=16$.) was $26.04 \% \pm 3.63 \%$. VER in aneurysms with residual neck (large-volume group, $n=25$; small-volume group, $n=53$ ) was $24.08 \% \pm 5.97 \%$. VER in residual aneurysms (large-volume group, $n=5$ ) was $18.36 \% \pm 4.60 \%$.

\section{Relationship between Use of Simple or Adjunctive Technique and VER}

Coiling by use of an adjunctive technique was performed in 54 aneurysms (balloon-assisted technique in 46 aneurysms, doublecatheter technique in 5 aneurysms, and both combined in 3 aneurysms). VER in cases by use of an adjunctive technique was $24.79 \% \pm 6.60 \%$, and VER in cases by use of a simple technique was $23.34 \% \pm 4.49 \%$. There was no statistically significant difference in VER between cases by use of an adjunctive or a simple technique ( $P=.41$, Mann-Whitney test).

\section{Relationship between Aneurysm Volume and VER and Its Impact on Outcome}

There were 31 aneurysms in the large-volume group, with a VER of $22.76 \% \pm 4.38 \%$. There were 69 aneurysms in the small-vol-
Table 4: Classification of follow-up angiography in each aneurysm group

\begin{tabular}{|c|c|c|}
\hline Aneurysm Group & Average & $\pm \mathrm{SD}$ \\
\hline \multicolumn{3}{|l|}{ Stable $(n=80)$} \\
\hline Height (mm) & 6.06 & 2.14 \\
\hline Width (mm) & 5.53 & 2.14 \\
\hline Neck (mm) & 5.04 & 1.87 \\
\hline Volume $\left(\mathrm{mm}^{3}\right)$ & 157.60 & 169.90 \\
\hline $\operatorname{VER}(\%)$ & 24.88 & 5.91 \\
\hline VER, large-volume $(n=19)$ & 23.84 & 4.28 \\
\hline VER, small-volume $(n=61)$ & 25.20 & 6.33 \\
\hline VER, adjunctive technique $(n=41)$ & 26.20 & 6.67 \\
\hline VER, simple technique $(n=39)$ & 23.50 & 4.70 \\
\hline \multicolumn{3}{|l|}{ Recanalization $(n=20)$} \\
\hline Height (mm) & 10.40 & 4.88 \\
\hline Width (mm) & 8.22 & 4.32 \\
\hline Neck (mm) & 7.23 & 2.88 \\
\hline Volume $\left(\mathrm{mm}^{3}\right)$ & 773.10 & 1014.00 \\
\hline VER (\%) & 21.10 & 3.85 \\
\hline VER, large-volume $(n=12)$ & 21.05 & 4.13 \\
\hline VER, small-volume $(n=8)$ & 21.18 & 3.64 \\
\hline VER, adjunctive technique $(n=12)$ & 20.36 & 4.03 \\
\hline VER, simple technique $(n=8)$ & 22.47 & 3.31 \\
\hline \multicolumn{3}{|l|}{ Re-treatment $(n=12)$} \\
\hline Height (mm) & 11.61 & 5.43 \\
\hline Width (mm) & 9.84 & 5.10 \\
\hline $\operatorname{Neck}(\mathrm{mm})$ & 8.23 & 2.83 \\
\hline Volume $\left(\mathrm{mm}^{3}\right)$ & 1081.00 & 1209.00 \\
\hline $\operatorname{VER}(\%)$ & 20.50 & 4.06 \\
\hline VER, large-volume $(n=9)$ & 20.67 & 4.46 \\
\hline VER, small-volume $(n=3)$ & 20.00 & 3.20 \\
\hline VER, adjunctive technique $(n=9)$ & 19.93 & 4.10 \\
\hline VER, simple technique $(n=3)$ & 22.20 & 4.18 \\
\hline
\end{tabular}

ume group, with a VER of $22.76 \% \pm 4.38 \%$. There was no statistically significant difference in VER between the large- and smallvolume groups ( $P=.12$, Mann-Whitney $U$ test).

In the large-volume group, 19 aneurysms were stable and had a VER of $23.84 \% \pm 4.28 \%$, and 12 aneurysms with recanalization had a VER of $21.05 \% \pm 4.13 \%$. There was no statistically significant difference in VER between stable and recanalized aneurysms in the large-volume group $(P=.11$ at $t$ test $)$.

Conversely, in the small-volume group, 61 aneurysms were stable and had a VER of $25.20 \% \pm 6.33 \%$, and 8 aneurysms with recanalization had a VER of $21.18 \% \pm 3.64 \%$. There was a statistically significant difference in VER between stable and recanalized lesions in the small-volume group $(P \leq .01$, Mann-Whitney $U$ test $)$.

\section{Relationship between Anatomic Follow-Up Results and VER}

Evaluation of anatomic outcomes at 1-year follow-up angiography showed the following: The stable group included 80 aneurysms, 55 unchanged and 25 with further thrombosis $(n=25)$; VER in the stable group was $24.88 \% \pm 5.91 \%$. The recanalization group (recanalization seen on follow-up angiography before retreatment decision) included 20 aneurysms with an overall VER of $21.10 \% \pm 3.85 \%$. Eight aneurysms in the recanalization group were only observed, as they had minor recurrences that were considered at low risk for rupture (and with a residual space too small to re-treat), and had a VER of $22.00 \% \pm 3.57 \%$. The re-treatment group included 12 aneurysms with a VER of $20.50 \% \pm 4.06 \%$ (Table 4). 


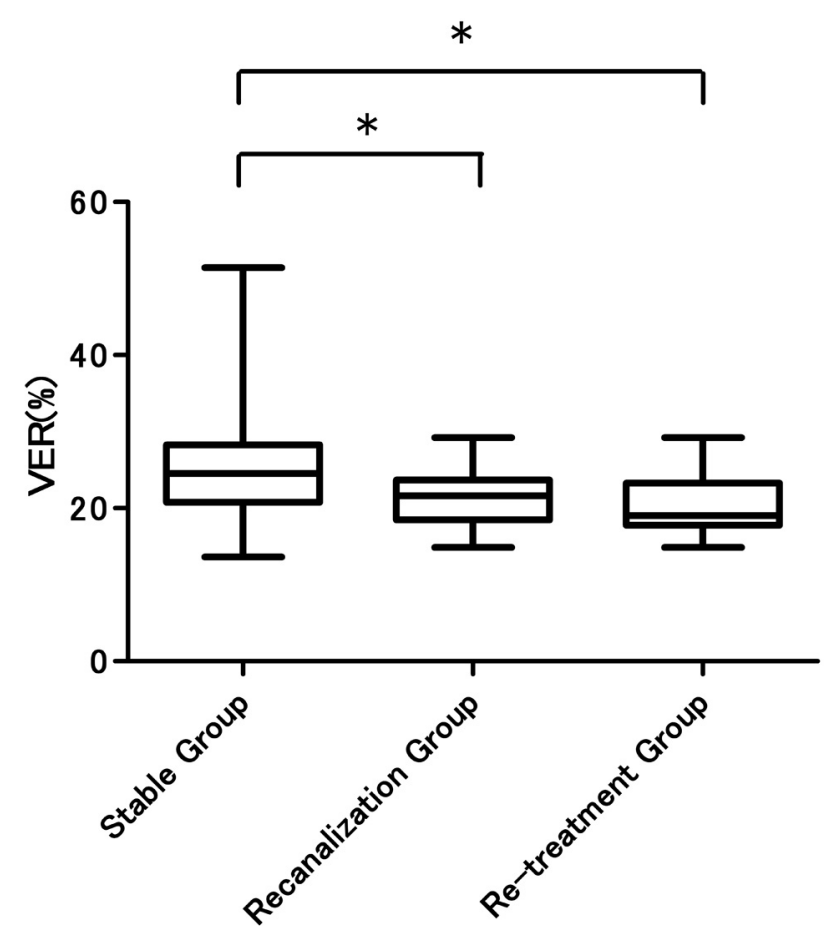

FIG 3. VER in each group ( ${ }^{\star}$ statistically significant difference, $P<.05$ ).

\section{Comparison of VER between Groups}

There was a statistically significant difference in VER between the stable group and the recanalization group under the Mann-Whitney $U$ test $(P \leq .01)$ and between the stable group and the retreatment group under the Mann-Whitney $U$ test $(\mathrm{P} \leq .01)$ (Fig 3).

We evaluated the cutoff points in VER between the stable group and the recanalization group by using ROC curve analysis. The optimal VER cutoff point for recanalization was $<25.40 \%$, at which the Youden index was 0.50 (sensitivity, 95.0\%; specificity, 42.5\%; area under the ROC curve, 0.70; 95\% CI, 0.58-0.81).

We also evaluated the cutoff points in VER between the stable group and the re-treatment group by using ROC curve analysis. The optimal VER cutoff point for re-treatment was $<19.15 \%$, at which the Youden index is 0.50 (sensitivity, 58.33\%; specificity, 87.50\%; area under the ROC curve, 0.74 ; 95\% CI, 0.59-0.88).

\section{DISCUSSION}

To date, there is no universally agreed-on approach to measure cerebral aneurysm dimensions. Differences in the measured values, when comparing modalities as well as the operating personnel, are found even among major studies. ${ }^{4,19-22}$ To address the need to standardize aneurysm measurements, Cybernet Systems developed an aneurysm measurement software, NV, for unbiased analysis. To our knowledge, there are no reports of similar software in the literature.

Recent work by Kuriyama et $\mathrm{al}^{23}$ has confirmed the accuracy of the NV software at measuring aneurysm size (neck length, height, and width), based on studies using a phantom model as well as 74 clinical cases using the size of the first framing coil chosen for embolization. Their study, however, did not evaluate the accuracy of NV volume measurements. Our limited in vitro validation by use of 4 silicone models showed that, compared with use of an AF, NV accurately measured aneurysm volume semiautomatically: Maximal percentage absolute error in aneurysm volume measurements by use of an $\mathrm{AF}$ was $45.63 \%$, which was higher than that achievable by NV (8.99\%).

The study by Kuriyama et $\mathrm{al}^{23}$ also did not evaluate the use of NV to calculate VER. VER has a strong relationship with aneurysm stability and is a useful predictor of aneurysm recanalization and re-treatment. An AF measurement may be very different from the actual aneurysm value, especially in the case of irregular aneurysm shapes, such as multilobulated aneurysms. In our study, the average AF volume was smaller than the ground truth value. Such volume measurement errors may result in a higher computed VER than the actual VER value, thereby giving the treating physician a false impression of achieving a safe treatment.

An in vitro study of VER by use of silicone sidewall aneurysms, which was published in 2000 , found that adequate embolization with platinum coils required maximal and minimal VER of $30 \%-$ $36 \%$ and $26 \%-33 \%$, respectively. ${ }^{24}$ Reports on the clinical application of VER and its impact on recanalization have indicated that values of $\geq 25 \%$ are necessary to achieve stability in aneurysms treated by endovascular embolization. ${ }^{6,7,9-11}$ We evaluated the VER cutoff point of aneurysm stability after endovascular embolization by using the ROC curve and the Youden index, ${ }^{17,18}$ and found a cutoff point of $25.40 \%$ in unstable aneurysms that is similar to values reported in the literature.

Recanalization alone does not imply the need for re-treatment. However, the cutoff point for re-treatment has not been previously reported in the literature. Our results showed an optimal VER cutoff point for re-treatment of $<19.15 \%$, which suggests that it is possible to re-treat cerebral aneurysms after coiling at a VER of $<20 \%$.

VER data have not yet been obtained for large or broad-neck aneurysms. ${ }^{6,9}$ Considering the specifics of the management of large and small aneurysms, we evaluated the difference between those $>7 \mathrm{~mm}$ in size and those smaller, in view of reported ${ }^{20}$ rupture risks. For this purpose, we chose a borderline aneurysm volume of $200 \mathrm{~mm}^{3}$ to separate large from small lesions. There was a statistically significant difference in VER between stable small-volume aneurysms (25.20\%) and recanalized small-volume aneurysms (21.18\%). Three small-volume aneurysms from the re-treatment group recanalized, all having low VER.

We also found no statistically significant difference in VER when using an adjunctive or a simple technique, with $24.79 \% \pm$ $6.60 \%$ (54 aneurysms) and $23.34 \% \pm 4.49 \%$ (46 aneurysms), respectively $(P=.41)$. The VER for recanalized aneurysms (recanalization group, $n=20)$ in the same 2 groups were $20.36 \% \pm$ $4.03 \%$ ( 13 aneurysms) and $22.47 \% \pm 3.31 \%$ (7 aneurysms), respectively. In some cases, the use of an adjunctive technique did not influence VER; in the stable group, the 41 aneurysms that were embolized by use of an adjunctive technique had a VER of $26.20 \% \pm 6.67 \%$, and the 39 aneurysms that were embolized with the simple technique had a VER of $23.50 \% \pm 4.70 \%(P=.08$, Mann-Whitney $U$ test).

Although VER is considered a valuable predictor of aneurysm recanalization, this has yet to be verified in a controlled, prospective, multicenter study. The NV volume measurement software may be useful in such a study.

AJNR Am J Neuroradiol 35:721-26 Apr 2014 www.ajnr.org 725 


\section{Possibility of Use of Bioactive Coils and Hydrogel-Coated Coils}

The development of bioactive coils promises further improvement in the long-term outcome of patients with aneurysms. ${ }^{25}$ With these coils, aneurysm occlusion stability may be achievable even at VER of $<25 \%$, and even large aneurysms may become candidates for successful embolization. Watanabe et $\mathrm{al}^{26}$ have reported the hydrogel-coated coil as being a safe and feasible device for improving the packing efficacy in endovascular coil embolization.

\section{Study Limitations}

The major limitation of our study was that it summarized data from a single center with a small number of patients treated and limited validation data from silicone phantom models. We need a multicenter, prospective cohort study with a large number of patients in which to conduct an evaluation of other detachable coils. A multicenter collaborative investigation of the NV software for the evaluation of recanalization of cerebral aneurysms after coiling is ongoing in Japan. As a next stage, we are considering the possibility of launching an international cooperative study.

\section{CONCLUSIONS}

The NV software provided accurate aneurysm volume measurements, which suggests that NV may be useful as a standardized tool to measure aneurysm size and volume, especially for conducting multicenter clinical studies. In addition to angiographic assessment, measurement of VER may be useful to predict future angiographic changes of aneurysm occlusion.

Disclosures: Toshihiaro Ishibashi-UNRELATED: Consultancy: Stryker; Payment for Lectures (including service on speakers bureaus): Stryker. Yuichi Murayama-UNRELATED: Consultancy: Stryker, Asahi INTECC, BrainLab; Payment for Development of Educational Presentations: Covidien, Comments Training course director; Grants/Grants Pending: Stryker, ${ }^{*}$ Siemens, ${ }^{*}$ FujiFilm*; Payment for Lectures (including service on speaker bureaus): Stryker, Terumo; Patents (planned, pending, or issued): Stryker*; Royalties: Stryker*; Other Relationships: Cybernet: We requested Cybernet develop NeuroVision software without payment; Cybernet decided to provide software license to our institution without any financial requirement. *Money paid to institution.

\section{REFERENCES}

1. Gonzalez N, Murayama Y, Nien YL, et al. Treatment of unruptured aneurysms with GDCs: clinical experience with 247 aneurysms. AJNR Am J Neuroradiol 2004;25:577-83

2. Guglielmi G, Viñuela F, Sepetka I, et al. Electrothrombosis of saccular aneurysms via endovascular approach. Part 1: electrochemical basis, technique, and experimental results. J Neurosurg 1991;75:1-7

3. Murayama Y, Nien YL, Duckwiler G, et al. Guglielmi detachable coil embolization of cerebral aneurysms: 11 years' experience. J Neurosurg 2003;98:959-66

4. Wiebers DO, Whisnant JP, Huston J 3rd, et al. Unruptured intracranial aneurysms: natural history, clinical outcome, and risks of surgical and endovascular treatment. Lancet 2003;362:103-10

5. Hayakawa M, Murayama Y, Duckwiler GR, et al. Natural history of the neck remnant of a cerebral aneurysm treated with the Guglielmi detachable coil system. J Neurosurg 2000;93:561-68

6. Kawanabe Y, Sadato A, Taki W, et al. Endovascular occlusion of intracranial aneurysms with Guglielmi detachable coils: correlation between coil packing density and coil compaction. Acta Neurochir (Wien) 2001;143:451-55

7. Satoh K, Satomi J, Matsubara S, et al. Measurement of volume ratio to predict coil compaction, on aneurysmal embolization. Interv Neuroradiol 1998;4 Suppl 1:179-82

8. Szikora I, Wakhloo AK, Guterman LR, et al. Initial experience with collagen-filled Guglielmi detachable coils for endovascular treatment of experimental aneurysms. AJNR Am J Neuroradiol 1997;18:667-72

9. Tamatani S, Ito $\mathrm{Y}$, Abe H, et al. Evaluation of the stability of aneurysms after embolization using detachable coils: correlation between stability of aneurysms and embolized volume of aneurysms. AJNR Am J Neuroradiol 2002;23:762-67

10. Uchiyama N, Kida S, Nomura M, et al. Significance of volume embolization ratio as a predictor of recanalization on endovascular treatment of cerebral aneurysms with Guglielmi detachable coils. Interv Neuroradiol 2000;6 Suppl 1:59-63

11. Yagi K, Satoh K, Satomi J, et al. Evaluation of aneurysm stability after endovascular embolization with Guglielmi detachable coils: correlation between long-term stability and volume embolization ratio. Neurol Med Chir (Tokyo) 2005;45:561-65; discussion 565-66

12. Takao H, Murayama Y, Ishibashi T, et al. Comparing accuracy of cerebral aneurysm size measurements from three routine investigations: computed tomography, magnetic resonance imaging, and digital subtraction angiography. Neurol Med Chir (Tokyo) 2010;50:893-99

13. Otsu N. A threshold selection method from gray-level histograms. IEEE Trans Sys Man Cyber 1979;9:62-66

14. Vallée JN, Pierot L, Mont'alverne F, et al. Unruptured intracranial aneurysms treated by three-dimensional coil embolization: evaluation of the postoperative aneurysm occlusion volume. Neuroradiology 2005;47:438-45

15. Raymond J, Guilbert F, Weill A, et al. Long-term angiographic recurrences after selective endovascular treatment of aneurysms with detachable coils. Stroke 2003;34:1398-403

16. Roy D, Milot G, Raymond J. Endovascular treatment of unruptured aneurysms. Stroke 2001;32:1998-2004

17. Fluss R, Faraggi D, Reiser B. Estimation of the Youden Index and its associated cutoff point. Biom J 2005;47:458-72

18. Perkins NJ, Schisterman EF. The inconsistency of "optimal" cutpoints obtained using two criteria based on the receiver operating characteristic curve. Am J Epidemiol 2006;163:670-75

19. International Study of Unruptured Intracranial Aneurysms Investigators. Unruptured intracranial aneurysms-risk of rupture and risks of surgical intervention. $N$ Engl J Med 1998;339:1725-33

20. UCAS Japan Investigators, Morita A, Kirino T, et al. The natural course of unruptured cerebral aneurysms in a Japanese cohort. N Engl J Med 2012;366:2474-82

21. Molyneux AJ, Kerr RS, Yu LM, et al. International subarachnoid aneurysm trial (ISAT) of neurosurgical clipping versus endovascular coiling in 2143 patients with ruptured intracranial aneurysms: a randomised comparison of effects on survival, dependency, seizures, rebleeding, subgroups, and aneurysm occlusion. Lancet 2005;366: $809-17$

22. Sonobe M, Yamazaki T, Yonekura M, et al. Small unruptured intracranial aneurysm verification study: SUAVe study, Japan. Stroke 2010;41:1969-77

23. Kuriyama T, Furukawa H, Shimizu K, et al. [Importance of an automatic measurement base on the first coil diameter in regard to coil embolization of a cerebral aneurysm]. Nihon Hoshasen Gijutsu Gakkai Zasshi 2012;68:95-102 [article in Japanese]

24. Piotin M, Mandai S, Murphy KJ, et al. Dense packing of cerebral aneurysms: an in vitro study with detachable platinum coils. AJNR Am J Neuroradiol 2000;21:757-60

25. Murayama Y, Tateshima S, Gonzalez NR, et al. Matrix and bioabsorbable polymeric coils accelerate healing of intracranial aneurysms: long-term experimental study. Stroke 2003;34:2031-37

26. Watanabe K, Sugiu K, Tokunaga K, et al. Packing efficacy of HydroCoil embolic system: in vitro study using ruptured aneurysm model. Neurosurg Rev 2007;30:127-30; discussion 130 